\title{
USING VIRTUAL ENVIRONMENTS TO ASSESS TIME-TO-CONTACT JUDGMENTS FROM PEDESTRIAN VIEWPOINTS
}

By

\author{
A. Elizabeth Seward \\ Thesis \\ Submitted to the Faculty of the \\ Graduate School of Vanderbilt University \\ in partial fulfillment of the requirements \\ for the degree of \\ MASTER OF SCIENCE \\ in \\ Computer Science \\ May, 2007 \\ Nashville, Tennessee
}

Approved:

Robert E. Bodenheimer, Jr.

Julie A. Adams 


\section{ACKNOWLEDGMENTS}

I would like to thank Bobby Bodenheimer and Dan Ashmead, who acted as my advisors for the work presented in this thesis. This research was supported by the National Eye Institute, National Institutes of Health, under R01 EY12894-04 and by the National Science Foundation under IIS-0237621. 
TABLE OF CONTENTS

Page

LIST OF FIGURES $\ldots \ldots \ldots \ldots \ldots \ldots \ldots \ldots \ldots \ldots$ vi

LIST OF TABLES $\ldots \ldots \ldots \ldots \ldots \ldots \ldots \ldots \ldots \ldots \ldots \ldots \ldots$ viii

\section{Chapter}

I INTRODUCTION $\ldots \ldots \ldots \ldots \ldots \ldots \ldots \ldots \ldots \ldots \ldots$

II BACKGROUND $\ldots \ldots \ldots \ldots \ldots \ldots \ldots \ldots \ldots \ldots \ldots \ldots$

II.1 Types of Time-to-Contact Experiments . . . . . . . . . . . . . . . . . 4

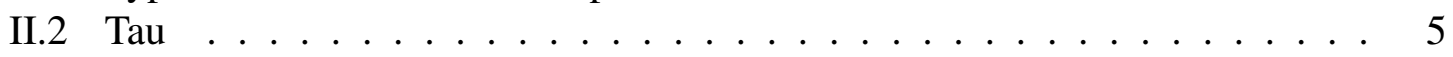

II.3 Influences on TTC Judgments $\ldots \ldots \ldots \ldots \ldots$

III EXPERIMENT 1: EFFECT OF VEHICLE MODEL $\ldots \ldots \ldots \ldots$

III.1 Method . . . . . . . . . . . . . . . . . . . . . . . 10

III.1.1 Subjects . . . . . . . . . . . . . . . . . . . . 10

III.1.2 Materials and Apparatus . . . . . . . . . . . . . . . 10

III.1.3 Procedure . . . . . . . . . . . . . . . . . . . . . . . . . 13

III.2 Results . . . . . . . . . . . . . . . . . . . . . . . . 16

IV EXPERIMENT 2: EFFECT OF PEDESTRIAN VIEWPOINT $\ldots \ldots \ldots$

IV.1 Method . . . . . . . . . . . . . . . . . . . . 20

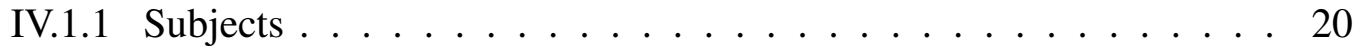

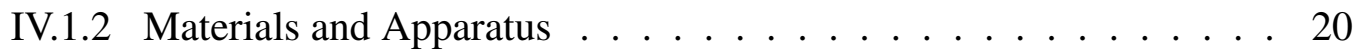

IV.1.3 Procedure . . . . . . . . . . . . . . . . . . . . 21

IV.2 Results . . . . . . . . . . . . . . . . . . . 23

V EXPERIMENT 3: SHORT- AND LONG-RANGE TTC JUDGMENTS ‥ 25

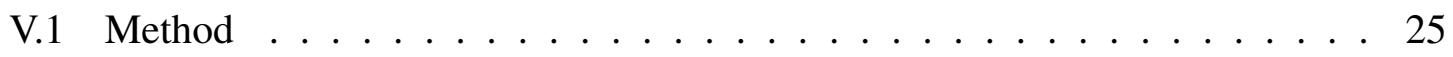

V.1.1 Subjects . . . . . . . . . . . . . . . 25

V.1.2 Procedures for Experiment 3a: Discrimination Task . . . . . . . 26

V.1.3 Procedures for Experiment 3b: Estimation Task . . . . . . . . . . 26

V.2 Results . . . . . . . . . . . . . . . . . . 26 
VI EXPERIMENT 4: EFFECT OF IMMERSIVE VIEWING . . . . . . . . 31

VI.1 Method ......................... . . 31

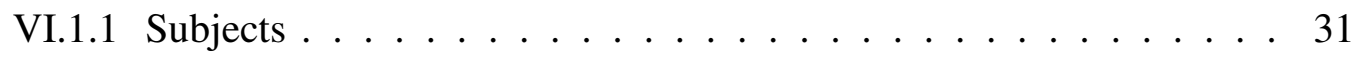

VI.1.2 Materials and Apparatus . . . . . . . . . . . . . 31

VI.1.3 Procedure . . . . . . . . . . . . . . . . 32

VI.2 Results ........................ 33

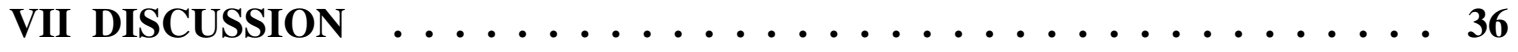

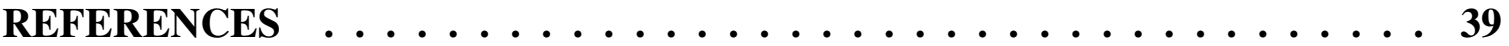




\section{LIST OF FIGURES}

Figure

III.1 Examples of the first image in a $2 \mathrm{~s}$ video for the lowest (10mph) and highest (30mph) velocities for each of the three reference values of TTC: $4 \mathrm{~s}, 7 \mathrm{~s}$, and 10s. The sedan model is shown in the $10 \mathrm{mph}$ images, and the truck model is shown in the $30 \mathrm{mph}$ images. . . . . . . . . . . . . . . . . . . 12

III.2 The enclosure used for experiments performed in a desktop environment. . 13

III.3 An example adaptive track following the two-one up-down staircase procedure for a single test subject. We measured the discrimination threshold by averaging the values of the last four reversals that the subject had. . . . . 15

III.4 Starting distance versus velocity for two reference TTC times, $7 \mathrm{~s}$ and $7.75 \mathrm{~s}$. In our procedure, if a subject was asked to discriminate between TTCs of $7 \mathrm{~s}$ and $7.75 \mathrm{~s}$, one of the black dots (7s TTC) would be randomly selected and one of the the yellow dots (7.75s TTC) would also be randomly selected. Since image size is directly proportional to starting distance, image size is decoupled from the TTC. . . . . . . . . . . . . . . . 16

III.5 The mean thresholds for the vehicle model discrimination task (Experiment 1). The means were determined by averaging the final four reversals for TTC reference times of $4 \mathrm{~s}, 7 \mathrm{~s}$, and $10 \mathrm{~s}$ for each vehicle model. Error bars show standard errors of the mean. . . . . . . . . . . . . . . . 18

III.6 The mean relative thresholds for the vehicle model discrimination task (Experiment 1) as functions of the TTC reference values. Error bars show standard errors of the mean. . . . . . . . . . . . . . . . . . . . . . 19

IV.1 Examples of the first image in a $2 \mathrm{~s}$ video for the the sedan, SUV, and truck from various viewpoints with a TTC of $4 \mathrm{~s}$ and a velocity of $20 \mathrm{mph}$. . . . . 21

IV.2 The mean thresholds for the viewpoint discrimination task (Experiment 2). The means were determined by averaging the final four reversals for TTC reference times of $6 \mathrm{~s}$ and 10s for each viewpoint. Error bars show standard errors of the mean. . . . . . . . . . . . . . . . . . . . . 24 
V.1 The mean thresholds for the discrimination task (Experiment 3a). The means were determined by averaging the final four reversals for TTC reference times of $4 \mathrm{~s}, 7 \mathrm{~s}$, and $10 \mathrm{~s}$. Error bars show standard errors of the mean. . . . . . . . . . . . . . . . . . 28

V.2 The mean relative thresholds for the discrimination task (Experiment 3a) as functions of the TTC reference values. Error bars show standard errors of the mean. . . . . . . . . . . . . . . . . . . . . . 28

V.3 The mean values for the estimation task (Experiment 3b). The means were determined by averaging each subject's mean estimated TTC for each reference value. Error bars show standard errors of the mean. The black solid lines show the correct estimations. . . . . . . . . . . . . . 30

VI.1 The mean thresholds for the viewpoint discrimination task in the immersive virtual environment (Experiment 4). The means were determined by averaging the final four reversals for TTC reference times of $4 \mathrm{~s}$ and $7 \mathrm{~s}$ for each viewpoint. Error bars show standard errors of the mean. . . . . . . . 35

VI.2 The mean relative thresholds for the vehicle model discrimination task in the immersive virtual environment (Experiment 4) as functions of the TTC reference values. Error bars show standard errors of the mean. . . . . . . . 35 


\section{LIST OF TABLES}

Table

III.1 Discrimination thresholds for each subject by TTC reference for the sedan model (Experiment 1). These values were computed by subject as the average of the last four reversals. Also shown are the population mean and standard error of the mean. . . . . . . . . . . . . . . . . . . . 17

III.2 Discrimination thresholds for each subject by TTC reference for the truck model (Experiment 1). These values were computed by subject as the average of the last four reversals. Also shown are the population mean and standard error of the mean. . . . . . . . . . . . . . . . . 18

IV.1 Discrimination thresholds for each viewpoint for the 6s TTC reference (Experiment 2). These values were computed by subject as the average of the last four reversals. Also shown are the population mean and standard error of the mean. . . . . . . . . . . . . . . . . . . . . 22

IV.2 Discrimination thresholds for each viewpoint for the 10s TTC reference (Experiment 2). These values were computed by subject as the average of the last four reversals. Also shown are the population mean and standard error of the mean. . . . . . . . . . . . . . . . . . 24

V.1 Discrimination thresholds for each subject by TTC reference for the discrimination task (Experiment 3a). These values were computed by subject as the average of the last four reversals. Also shown are the population mean and standard error of the mean. . . . . . . . . . . . . . 27

V.2 Results from Experiment 3b. For each subject their mean estimated TTC for each reference value with their standard error over 10 trials shown in parentheses. Additionally, for each subject the slope of a linear regression of their data is shown in the final column. The total mean estimated value and average slope are shown in the bottom row. . . . . . . . . . . 30

VI.1 Discrimination thresholds for each viewpoint for the $4 \mathrm{~s}$ TTC reference (Experiment 4). These values were computed by subject as the average of the last four reversals. Also shown are the population mean and standard error of the mean. . . . . . . . . . . . . . . . . . 34 
VI.2 Discrimination thresholds for each viewpoint for the 7s TTC reference (Experiment 4). These values were computed by subject as the average of the last four reversals. Also shown are the population mean and standard error of the mean. . . . . . . . . . . . . . . . . . . . . . 34 


\section{CHAPTER I}

\section{INTRODUCTION}

An important application of computer graphics is the creation of sufficiently realistic simulations that provide the ability to analyze and learn about real-world situations that are otherwise difficult and expensive to reproduce. One of these situations involves understanding pedestrian behavior at street crossings. In 2003, motor vehicle accidents were the leading cause of death in the U.S. for persons between the ages of 4 and 33. A significant number of motor vehicle accidents every year involve pedestrians. In 2005, over 4800 pedestrians were killed as a result of traffic accidents, and an additional 64,000 were in-

jured [National Center for Statistics and Analysis 2005]. Crossing the street is a commonly encountered situation, yet, as is evident from the accident statistics, pedestrians are making poor decisions about when to cross.

To address this problem, we would like to be able to identify perceptual capacities that affect pedestrians' access to information about traffic and street crossings. Many perceptual cues, both auditory and visual, are involved in street-crossing decisions. Visual cues, such as perceived distance, arrival time of approaching vehicles, and the relative sizes of other surrounding objects are all potential factors that may influence street-crossing judgments. Studying these capabilities in real pedestrian crossings is difficult due to the number of factors that must be controlled in a large outdoor environment. For example, not only do we require a large outside testing space, but also exact distance and speed measurements for vehicles, and suitable weather conditions. Our goal is to use virtual simulations to examine and learn about pedestrian perceptions of traffic. In particular, we would like to establish that people's perceptual capacities allow them to reason about the arrival time, or time-tocontact (TTC), of approaching vehicles in the real world similarly when they are presented graphically in desktop and immersive virtual environments. Ultimately, these findings may 
aid in the design of better street crossings in the future. This thesis lays a groundwork in the area of pedestrian street crossings with these goals in mind.

This work presents the results of a set of experiments designed to assess the ability of people to discriminate and estimate TTC for approaching vehicles in a virtual environment under a variety of conditions. These conditions include analyzing whether such factors as viewpoint (side of street), type of car (e.g., sedan versus semi-trailer truck), and presentation mode (desktop environment versus head-mounted display) affect people's ability to discriminate TTC. Much of the literature on TTC, e.g., [Todd 1981; Gray and Regan 1999; DeLucia and Novak 1997], uses short TTC reference values (e.g., 4s) in assessing people's discriminative ability. Pedestrians, however, typically make crossing decisions for TTCs that are significantly longer. Ashmead et al. [2005] and Guth et al. [2005] investigated realworld decisions to cross the street at traffic roundabouts and found that pedestrians had to choose gaps in traffic of 6 s or more. Since little literature exists on people's ability to discriminate and estimate TTCs at these longer ranges, we want to examine such judgments in a controlled setting. Our experiments are conducted over both short and long TTCs; the short ranges provide useful comparison with the existing literature, and the long ranges assess how variable discrimination ability is as the TTC reference changes.

In particular, this thesis describes four experiments, all focusing on understanding influences on TTC judgments in pedestrian street-crossing situations. Experiment 1 examines the effect of vehicle model on TTC judgments for two different types of vehicles, a sedan and a semi-trailer truck. Experiment 2 looks at different pedestrian viewpoints and their influence on people's ability to judge TTC. Experiment 3 investigates discrimination and estimation thresholds for desktop simulations and compares these findings to other similar tasks in the TTC literature, e.g., [Schiff and Detwiler 1979; Cavallo and Laurent 1988]. Finally, Experiment 4 examines the effect of self-motion on TTC judgments in an immersive virtual environment. We find that both vehicle model and pedestrian viewpoint do not affect TTC judgments. In addition, TTC judgments seem to be unaltered by the addition of 
self-motion in an immersive virtual environment. Also, our results for short TTCs are consistent with those in the literature. For longer TTCs that are important for traffic-crossing decisions, however, we observe a gradual decline in TTC discrimination accuracy.

Moreover, this thesis presents a system designed to test various perceptual factors. Perception is an important aspect of computer graphics and animation because it provides a performance metric by which to judge the quality of representation. This thesis assesses these representations by comparing TTC judgments in virtual environments to published data for similar judgments made in the real world.

This thesis is organized as follows. Chapter II discusses related work involving TTC studies. Chapters III-VI describe the above mentioned experiments and their results. We conclude with a discussion of them in Chapter VII. 


\section{CHAPTER II}

\section{BACKGROUND}

This chapter describes related work about time-to-contact. Included are types of experiments and environments that have typically been used to study time-to-contact. Also, several influences on TTC judgments are described.

\section{II.1 Types of Time-to-Contact Experiments}

TTC is the time remaining until a moving object reaches an observer or a particular location. TTC has also been termed time-to-go, time-to-coincidence, time-to-collision, timeto-arrival, and time-to-passage [Caird and Hancock 1994]. TTC research can be divided into two main categories according to the methodology employed in the experiments. One class of experiments, termed coincidence-anticipation tasks, involves having subjects make estimations as to when a target will reach a given position [Tresilian 1995]. One type of coincidence-anticipation tasks is prediction-motion tasks, in which the moving object is occluded from view before it reaches the observer or specified point. The subject then indicates a response at the moment of the object's speculated arrival time [Tresilian 1995; Benguigui et al. 2003; Hecht et al. 2002]. Another subset of coincidence-anticipation tasks is experiments involving interceptive action tasks, such as catching or hitting balls [Caljouw et al. 2004; Senot et al. 2003; Servos and Goodale 1998; Tresilian 1993]. The second class of experiments includes relative judgment tasks, in which subjects distinguish between two or more different values of TTC [Tresilian 1995; DeLucia and Novak 1997; Todd 1981]. Each class represents a different approach to measuring how well people perceive TTC. In this work, we examine the correlation between these two approaches through performing relative judgment tasks and prediction-motion tasks.

For discrimination and estimation tasks, two important trends have generally been ac-

knowledged. The first is that discrimination thresholds for small TTC differences are usu- 
ally around 5-10\% [Todd 1981; Regan and Hamstra 1993]. The second trend is that people tend to have a conservative bias when estimating TTC [Schiff and Detwiler 1979; Cavallo and Laurent 1988; Gray and Regan 1999]. This bias means that they tend to underestimate TTC.

Although many experiments have been conducted in a laboratory setting, TTC judgments have also been studied in a more immersive environment. Plumert et al. [2004] examined how adults and children judged gaps in traffic and decisions to cross streets by riding a stationary bicycle through an immersive simulated environment. Caird and Hancock [1994] studied perception of oncoming traffic at an intersection using a driving simulator. Real-world experiments have also been conducted. Cavallo and Laurent [1988] observed TTC estimations for subjects riding as passengers in a car riding towards a stationary object. Kiefer et al. [2006] employed realistic rear-end crash situations to observe driver sensitivity to time-to-contact.

\section{II.2 Tau}

Many TTC studies aim to understand how people estimate TTC. Lee [1976] proposed that the means for obtaining the perceptual information necessary to estimate TTC is a quantity named $\tau$. It is defined as

$$
\tau=\frac{\theta}{\frac{d \theta}{d t}}
$$

where $\theta$ is the angle formed by the moving object with respect to the observer. Lee suggested that this cue is what people rely on to make a TTC judgment.

Several researchers have examined TTC judgments and found that people based their judgments on $\tau$. Schiff and Detwiler [1979] looked at whether people made TTC estimates based on two-dimensional information, i.e., $\tau$, or three-dimensional information, such as distance or distance-change, and found that optical $\tau$ was the best predictor for TTCs up to 10s. Gray and Regan [1999] also found that when they presented subjects with an expanding circle with different starting sizes and different TTCs, subjects based their estimations 
on $\tau$.

Others have claimed that Lee's $\tau$ may be unreliable in providing enough information about perceived TTCs, and that people do not respond to $\tau$ when judging TTC. Cavallo and Laurent [1988] assert that in real-world driving conditions, both speed and distance information are used to estimate TTC. Their results do not rule out the use of $\tau$ but demonstrate that $\tau$ alone cannot predict TTC judgments for this particular experiment. Smeets and Trébuchet [1996] claim that TTC judgments are best informed by perceived velocity. Several researchers have adopted the idea that Lee's $\tau$ may not be sufficient for determining how people judge TTC and that $\tau$ may be used in conjunction with other cues [DeLucia and Novak 1997; Tresilian 1995]. Other researchers have proposed alternate versions of $\tau$ [Bootsma and Craig 2002; Todd 1981].

Other cues other than $\tau$ have also been claimed to influence TTC decisions. Examples of these are image size, height in field, occlusion, and motion parallax [Heuer 1993; DeLucia 2004]. For our studies, we are not committed to whether $\tau$ or any other variable underlies or predicts TTC judgments. Our study focuses instead on how judgments vary according to the TTC range and overall influences on these judgments.

\section{II.3 Influences on TTC Judgments}

Some TTC work has examined whether binocular or monocular viewing of the stimuli affects TTC judgments. Cavallo and Laurent [1988] conducted an estimation task in which subjects riding as passengers in a car estimated the time-to-contact with a stationary object. They found that estimation accuracy was better for binocular vision than monocular vision at low speeds (around $19 \mathrm{mph}$ ) and nearer targets. Von Hofsten et al. [1992] performed an interceptive action task in which subjects caught balls and found that catching performance (whether the ball was caught) was better with binocular vision. In contrast, Servos and Goodale [1998] conducted a similar catching experiment and did not observe a difference in performance with binocular vision compared with monocular vision. They found that 
using binocular vision instead of monocular vision did not yield any differences in how long subjects took to reach the ball, the distance that the arm travelled before grasping the ball, or the distance the ball travelled before it was caught. They speculate that this difference may be attributed to the predictability of the ball trajectories in their task.

In a typical street crossing situation, gaps in traffic during which it is safe to cross correspond to cars with TTCs around 7s-10s. Schiff and Detwiler [1979] examined TTC judgments at these longer TTCs. They studied TTC estimation for films of a black square moving toward the observer. For TTCs of $4 \mathrm{~s}, 6 \mathrm{~s}, 8 \mathrm{~s}$, and 10s, they found a significant effect of time, meaning that people's ability to estimate TTC decreased significantly as the TTC value increased. Using films of vehicles approaching a camera, Schiff and Oldak [1990] found that TTC estimation accuracy decreased as the TTC increased from $1.5 \mathrm{~s}$ to $6 \mathrm{~s}$. McLeod and Ross [1983] used films that were recorded from the passenger seat of a car with TTCs of 3.6s, 4.5s, 6s, and 9s. Estimation accuracy was found to decrease as the TTC value increased.

One of the factors that may influence TTC judgments is the type of presented stimulus or its size. Schiff and Detwiler [1979] compared TTC judgments for films showing a black form, either 3 or $12 \mathrm{~cm}$ in diameter, and found no effect of object size. These researchers also examined the relationship between TTC judgments for films with black forms and films showing real automobiles. Again, they found no significant difference. Kim and Grocki [2006] performed an experiment with TTC estimation for animated polyhedrons, either $1.8 \times 1.6 \times 1.5$ meters or $0.6 \times 1.0 \times 2.8$ meters, and also found no effect of object size. Bootsma and Oudejans [1993] conducted a discrimination task involving squares with sides of either 8.5 or 9.5 centimeters, and found that the size of the objects did not influence subjects' discrimination ability. On the other hand, Caird and Hancock [1994] observed an effect of object size or stimulus type. Using a driving simulator, they observed TTC estimations for four different vehicles approaching the observer at an intersection. Mean accuracy declined as the size of the car increased. Underestimations increased for 
larger vehicles. Therefore, mixed results have been found for the influence of stimulus type or size.

The trajectory of the stimulus in relation to the observer or the observer's viewpoint may also impact TTC judgments. Schiff and Oldak [1990] looked at arrival time judgments for films of cars with a head-on approach versus a bypass approach. The bypass angle for the films was less than $10^{\circ}$ at the vanishing point. No difference in estimation accuracy was found for head-on approaches compared with bypass paths. They performed a second experiment in which they observed TTC estimations for radial, transverse, and oblique trajectories using animated tabletop photography of a toy car. The radial path was a headon approach. In the transverse trajectory, the subject observed a side view of the car, in which the car moved left to right across the field of view. The oblique approach showed the car moving toward a target at a $45^{\circ}$ angle to the observer's viewpoint. Accuracy improved from the radial to the oblique to the transverse trajectories. Schiff and Oldak conclude that the further the trajectory from the radial direction, the more accurate the TTC judgment.

Manser and Hancock [1996] also studied the effect of approach angle. However, they observed the impact of the retinal center versus the retinal periphery on TTC judgments using computer-generated scenes projected onto a front and side wall. On the front wall, a car with head-on trajectory was shown. On the side wall, a car approaching on a road at a $40^{\circ}$ angle was displayed. Each trial showed one of the two trajectories. Subjects were instructed to look straight ahead at all times during the experiment. The $40^{\circ}$ angle approach was judged significantly less accurately than the head-on approach, which illustrates that the retinal periphery provides less information about TTC estimates than the retinal center.

For this work, we want to examine some of the above-mentioned influences on TTC judgments for pedestrian crossing situations. Chapter III describes an experiment to observe whether the type of vehicle model impacts TTC judgments. Chapter IV looks at the effect of common pedestrian viewpoints on TTC judgments. Chapter V observes shortrange versus long-range TTC judgments and compares a discrimination task and an es- 
timation task. Finally, Chapter VI presents an experiment observing the influence of an immersive virtual environment on TTC judgments. 


\section{CHAPTER III}

\section{EXPERIMENT 1: EFFECT OF VEHICLE MODEL}

This chapter presents an experiment to examine the influence of vehicle type on TTC judgments. The task and its results are described.

\section{III.1 Method}

To examine the effect of vehicle model on TTC judgments, we performed a study involving a discrimination task. Video animations of a graphical sedan or a semi-trailer truck moving down a road directly toward the viewer at different TTCs, velocities, and starting positions were used. We rendered the vehicles on a direct approach as opposed to the view that a person might have upon deciding whether to cross the street. We chose this option because it is the simplest situation and is consistent with approaches taken in other TTC studies, e.g., [Schiff and Detwiler 1979].

\section{III.1.1 Subjects}

Eight subjects, four males and four females, completed this study. Subjects' ages ranged from 23-52. All had normal or corrected-to-normal vision. Subjects were not compensated.

\section{III.1.2 Materials and Apparatus}

To apply TTC analyses directly to the application of pedestrian crossings using desktop and virtual environments, we used animated videos of a 3D graphical sedan model or semitrailer truck model travelling down a road towards the viewer for our stimuli. The rendered vehicle images were modelled to preserve real-world proportions and viewing conditions. Dimensions of the road followed guidelines in a 2005 Iowa Department of Transportation road specifications document [2005], and dimensions of the car and truck models were 
typical for vehicles of these kinds. We rendered images of the approaching vehicles at a resolution of $720 \times 480$. To light the scene, we used a single directional light in the direction of the camera. We rendered the scenes without shadows and without compression. Shadows can provide important depth cues, but for an outside scene, they would be time-ofday dependent. In the real world, for low to the ground objects like vehicles, shadows may provide an extra perceptual cue that our studies will not capture. To provide the clearest images possible and avoid compression artifacts, we generated uncompressed videos.

To represent the range of TTCs and velocities that pedestrians encounter in a typical street-crossing situation, $2 \mathrm{~s}$ videos were generated for TTCs from $2 \mathrm{~s}$ to $14 \mathrm{~s}$ at $0.25 \mathrm{~s}$ intervals. We define TTC to be the time remaining from the first frame of video until the time when the vehicle would reach the observer were it to continue its path at its current velocity. A given TTC was used in five separate videos, each with one of five constant vehicle velocities: $10,15,20,25$, and $30 \mathrm{mph}$. This range of vehicle speeds corresponds to settings where pedestrian activity is high [Pitt et al. 1990]. A velocity and a TTC value determined the starting distance of the vehicle in the first video frame. Two-second videos were used because this amount of time is probably about how long pedestrians take to decide whether there is a sufficient gap between vehicles to allow them to safely cross the street. In fact, varying viewing time between $2 \mathrm{~s}$ and $6 \mathrm{~s}$ in TTC experiments has been found to have no effect on the accuracy of TTC estimations [Sidaway et al. 1996; McLeod and Ross 1983]. To compare short- to long-range TTCs, we designed our experiments to be centered around three reference TTCs: $4 \mathrm{~s}, 7 \mathrm{~s}$, and 10s. Example images for the slowest and fastest velocities for these references are shown in Figure III.1. A blank (black) screen followed each animation to prevent the viewer from gaining any more visual information after the presentation of the stimulus.

To accurately represent the perspective of an observer, the observer's viewing angle was maintained in the rendered images. Therefore, for example, when the 6.5 -food-wide car was 440 feet away (a TTC of 10s at a velocity of $30 \mathrm{mph}$ ), the viewing angle would 


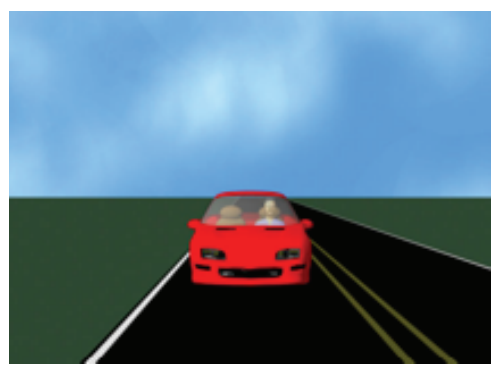

(a) $4 \mathrm{~s}$ at $10 \mathrm{mph}$.

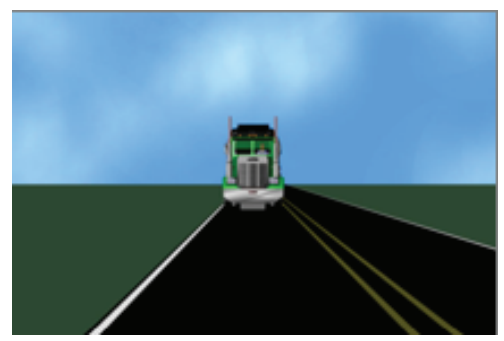

(d) $4 \mathrm{~s}$ at $30 \mathrm{mph}$.

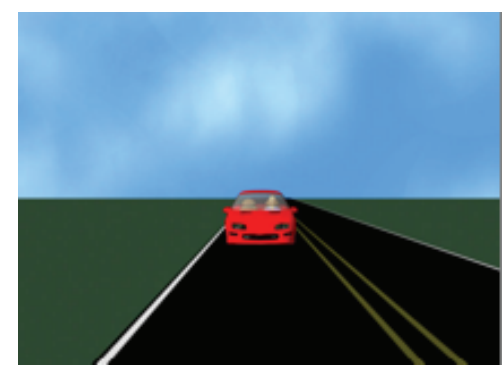

(b) $7 \mathrm{~s}$ at $10 \mathrm{mph}$.

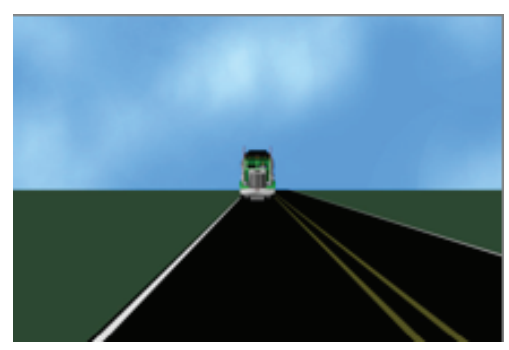

(e) $7 \mathrm{~s}$ at $30 \mathrm{mph}$.

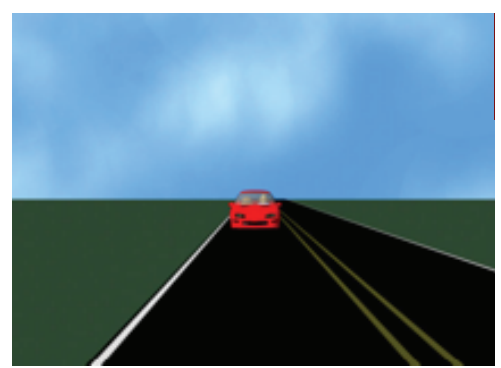

(c) $10 \mathrm{~s}$ at $10 \mathrm{mph}$.

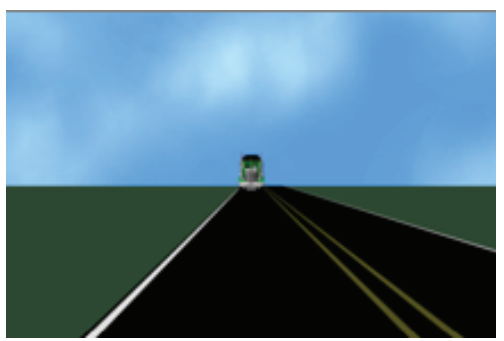

(f) $10 \mathrm{~s}$ at $30 \mathrm{mph}$.

Figure III.1: Examples of the first image in a 2 s video for the lowest (10mph) and highest (30mph) velocities for each of the three reference values of TTC: $4 \mathrm{~s}, 7 \mathrm{~s}$, and 10s. The sedan model is shown in the $10 \mathrm{mph}$ images, and the truck model is shown in the $30 \mathrm{mph}$ images.

be approximately $0.84^{\circ}$. We calibrated the sizes by assuming a viewing distance of two feet from the screen, and enforced it by requiring viewers to watch the videos through an enclosure. The enclosure, a black box, $10^{\prime \prime} \times 6.7^{\prime \prime} \times 24^{\prime \prime}$, was attached to the monitor such that it framed the videos, which were $10^{\prime \prime} \times 6.7^{\prime \prime}$. The viewing box also served to minimize distractions from the surrounding room. This enclosure is shown in Figure III.2.

We investigated whether binocular vision of a computer screen induced interfering signals for time-to-contact by conducting a small pilot study prior to these experiments. Six lab members were tested with a discrimination task similar to Experiment 1 described below. Each subject performed the experiment both with binocular and monocular vision. Monocular vision was obtained by having subjects wear an eye patch. All subjects performed equally well or better binocularly compared to monocularly. In addition, for the monocular vision portion of the task, some subjects complained of eyestrain and fatigue. We conjecture that binocular vision performance was better because the visual system is 


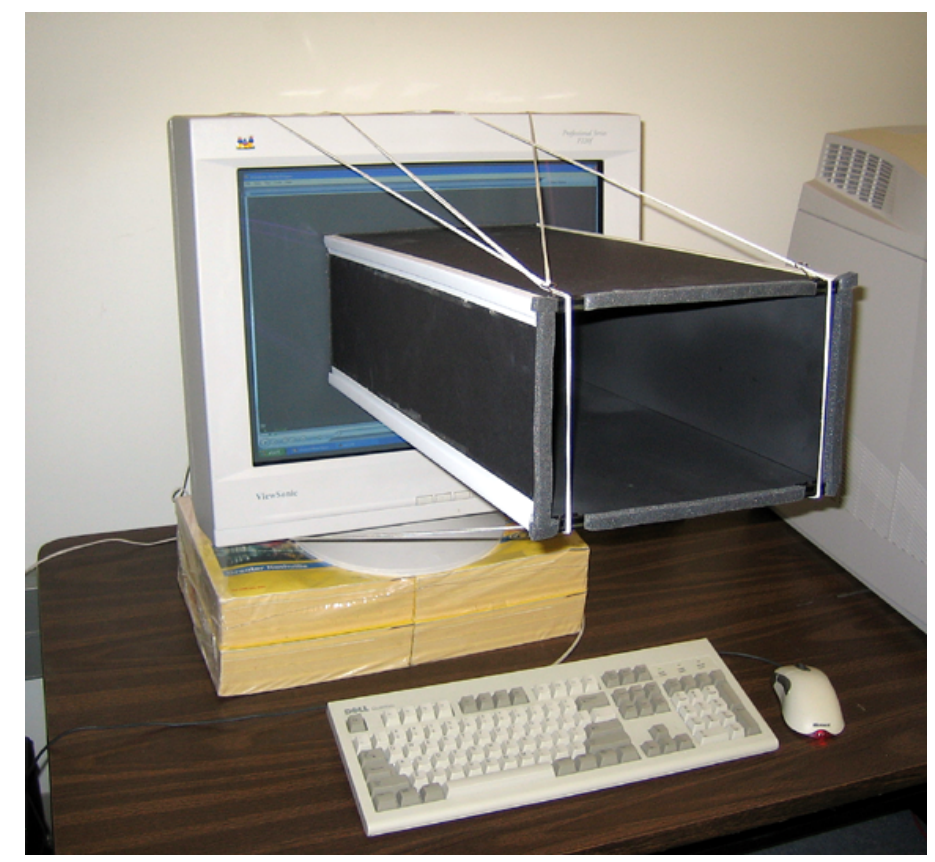

Figure III.2: The enclosure used for experiments performed in a desktop environment.

getting two opportunities to register the relevant visual cues, or it may be an effect of binocular summation. However, we did not pursue the question further. Thus, to provide comfortable testing conditions and also to measure subjects best possible TTC perceptions, all subjects in the experiments described in this thesis were tested binocularly.

\section{III.1.3 Procedure}

A discrimination task was conducted to test the effect of vehicle model on TTC judgments. Subjects were given a set of written instructions for the experiment and shown two practice trials. In a given trial, subjects were presented with a pair of $2 \mathrm{~s}$ videos and asked to determine which vehicle would reach them first. Each trial compared two videos showing the sedan or two videos showing the truck. The order of models presented was randomly selected. The videos were presented consecutively with a $2.5 \mathrm{~s}$ black screen between them. This amount of time seemed to give subjects just long enough to be ready to view the next video in the trial. Each pair of videos had a video with one of the three TTC reference 
values: 4s, 7s, or 10s, and another video with a TTC that was greater or less by an amount that varied across trials according to an adaptive threshold rule. The order of the pair of videos within each trial was random. To select which vehicle would reach them first, subjects pressed designated keys on a keyboard. Subjects were allowed to view a trial only once, and they were not provided any feedback from trial-to-trial.

Separate threshold estimates were obtained for each of the reference values of $4 \mathrm{~s}, 7 \mathrm{~s}$, and 10s for both the sedan and the truck, but these threshold procedures were interleaved, so that from a subject's perspective, a wide range of TTCs was presented. That is, there were six threshold procedures, one for each (Vehicle Model, TTC Reference) pair. Each threshold procedure was a staircase in which the difference between TTCs on a trial was decreased (made more difficult) after two consecutive correct trial outcomes, and increased (made less difficult) after an incorrect trial [Wetherill and Levitt 1965]. Increases and decreases in TTC differences were performed in $0.25 \mathrm{~s}$ increments. The experiment ended when the subjects reached 10 reversals or gave seven correct answers in a row for the lowest TTC difference, $0.25 \mathrm{~s}$, for both vehicle models and each TTC reference. A reversal consisted of an incorrect answer after a prior sequence of two consecutive correct answers or two consecutive correct answers after a sequence of incorrect and single correct answers (correct answers followed or preceded by an incorrect answer). An example track for one subject in the task is shown in Figure III.3. No subject in our experiment discriminated successfully seven times in a row at the $0.25 \mathrm{~s}$ difference. Our threshold procedures resulted in a variable number of trials for each (Vehicle Model, TTC reference) threshold pair. The number of trials was approximately the same across these conditions. The average number of trials per threshold procedure was 36.

The initial TTC difference between the videos for each TTC reference was $2 \mathrm{~s}$. For example, for a TTC reference of $4 \mathrm{~s}$, the initial trial for this reference would show a video of a vehicle with a TTC of $4 \mathrm{~s}$ at a random velocity and a vehicle with a TTC at either $2 \mathrm{~s}$ or $6 s$ at a random velocity. For the TTC reference of $4 \mathrm{~s}, 7 \mathrm{~s}$, and $10 \mathrm{~s}$, the maximum allowable 


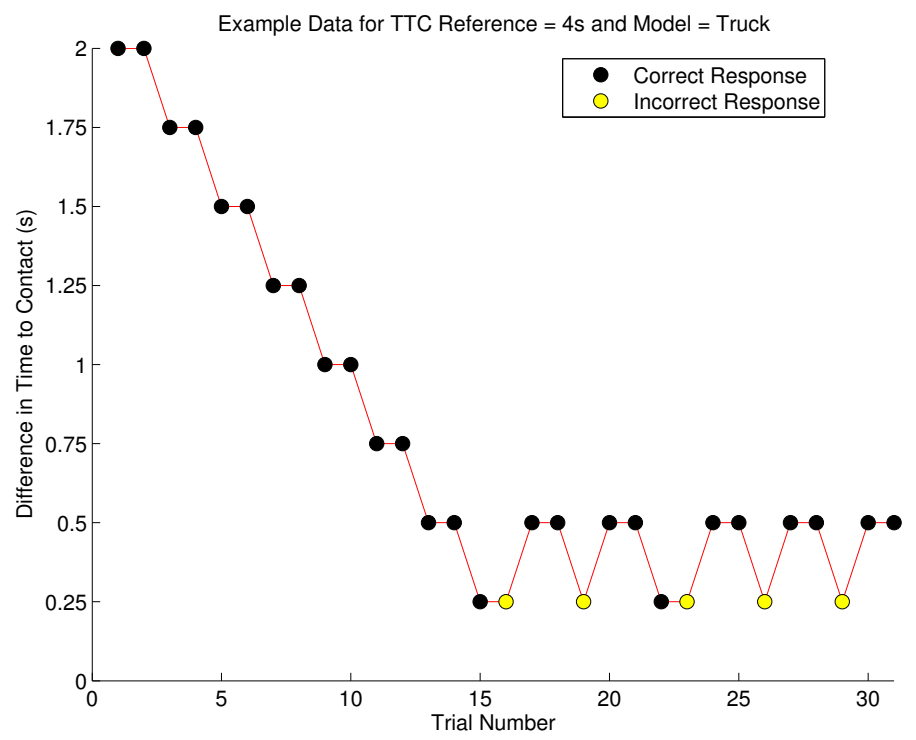

Figure III.3: An example adaptive track following the two-one up-down staircase procedure for a single test subject. We measured the discrimination threshold by averaging the values of the last four reversals that the subject had.

TTC differences were $2 \mathrm{~s}, 3 \mathrm{~s}$, and $4 \mathrm{~s}$ respectively. To discourage subjects from using only image size as a cue, one of five velocities $(10,15,20,25$, or $30 \mathrm{mph})$ was randomly selected for each video. We did not control for effects of velocity.

The videos were designed to match the ranges of vehicle speed and distance over which pedestrians typically have to make street crossing decisions. Over these ranges, the image size of a vehicle is not a reliable cue for approach time. For example, Figure III.4 shows the scenario for a trial in the discrimination task in which the two videos had TTC values of 7s and 7.75s. The black dots show the five videos that were available for the $7 \mathrm{~s}$ TTC, one of which was randomly chosen by the computer. Likewise, the computer randomly selected from the five available videos for the 7.75s TTC. Given that both videos were selected randomly across a range of starting distance from about 100-300 feet, and that image size is directly proportional to starting distance, image size was strongly decoupled from the TTC. 


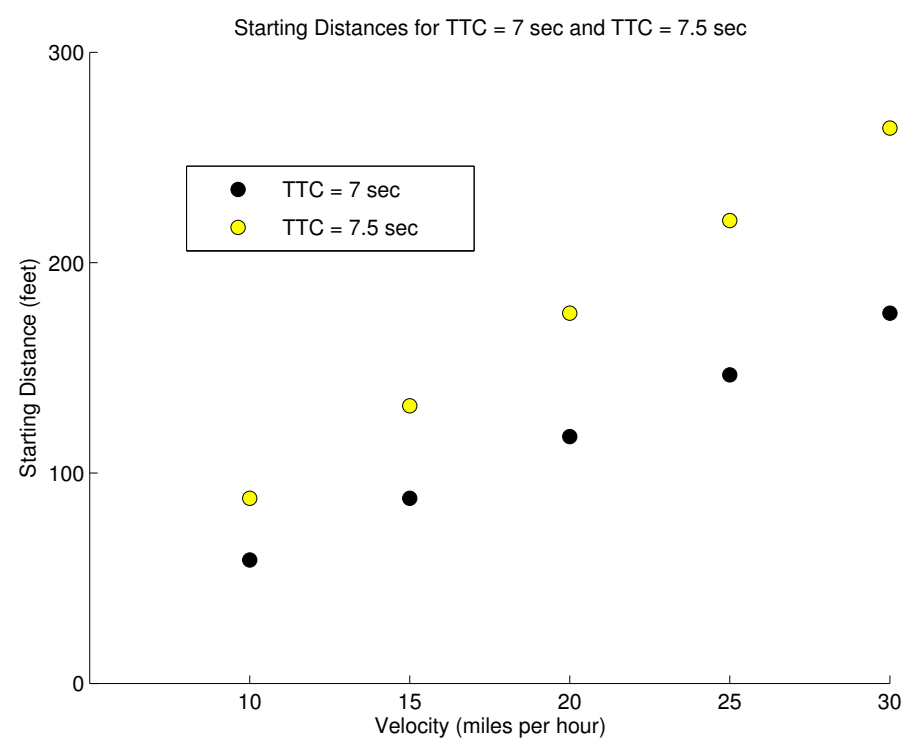

Figure III.4: Starting distance versus velocity for two reference TTC times, $7 \mathrm{~s}$ and 7.75s. In our procedure, if a subject was asked to discriminate between TTCs of $7 \mathrm{~s}$ and $7.75 \mathrm{~s}$, one of the black dots (7s TTC) would be randomly selected and one of the the yellow dots (7.75s TTC) would also be randomly selected. Since image size is directly proportional to starting distance, image size is decoupled from the TTC.

\section{III.2 Results}

Tables III.1 and III.2 show the discrimination thresholds for each participant that were computed as the average of the TTC differences at the last four reversals. These are also shown in Figure III.5. A trend analysis of variance was performed with both TTC Reference $(4,7,10)$ and Vehicle Model as repeated-measures factors. Trend analysis was used since the independent variable TTC Reference is on a quantitative, ordered measurement scale [Maxwell and Delaney 2004]. This test allowed us to see how TTC discrimination ability changed with respect to the TTC Reference value. There was a significant linear trend for TTC References, $F(2,14)=44.928, p<.001$. Neither Vehicle Model nor the interaction effect was significant.

Discrimination thresholds were also converted from seconds to a proportion of the reference TTC to assess whether discrimination ability is a constant proportion of the refer- 


\begin{tabular}{|c|l|l|l|}
\hline & \multicolumn{3}{|c|}{ Sedan } \\
\hline Subject & TTC Ref. =4s & TTC Ref. =7s & TTC Ref. = 10s \\
\hline 1 & 0.56 & 0.44 & 2.19 \\
\hline 2 & 0.69 & 0.5 & 2.56 \\
\hline 3 & 0.38 & 0.44 & 2.63 \\
\hline 4 & 0.5 & 1.44 & 2.88 \\
\hline 5 & 0.44 & 1.25 & 1.63 \\
\hline 6 & 0.38 & 0.94 & 3.19 \\
\hline 7 & 0.56 & 1.38 & 1.44 \\
\hline 8 & 0.38 & 1.38 & 2.13 \\
\hline Means (Std. Error) & $0.49(0.11)$ & $0.97(0.45)$ & $2.32(0.60)$ \\
\hline
\end{tabular}

Table III.1: Discrimination thresholds for each subject by TTC reference for the sedan model (Experiment 1). These values were computed by subject as the average of the last four reversals. Also shown are the population mean and standard error of the mean.

ence value of TTC. For example, a threshold of $0.8 \mathrm{~s}$ in the $4 \mathrm{~s}$ sedan condition would be converted to $(0.8) / 4=0.2$. Mean relative thresholds for the sedan model were $0.12,0.14$, and 0.23 of the 4, 7, and 10 reference values, respectively. Mean relative thresholds for the truck model were $0.13,0.16,0.18$ of the 4,7 , and 10 reference values, respectively. These relative thresholds are shown in Figure III.6. They were analyzed with the same design including TTC Reference and Vehicle Model. Again, only the linear trend for TTC Reference was significant, $F(2,14)=9.076, p=.003$. Therefore, the type of vehicle model did not significantly affect TTC judgments, and discrimination accuracy decreased absolutely and relatively for longer TTCs across vehicle models. 


\begin{tabular}{|c|l|l|l|}
\hline & \multicolumn{3}{|c|}{ Truck } \\
\hline Subject & TTC Ref. =4s & TTC Ref. =7s & TTC Ref. = 10s \\
\hline 1 & 0.375 & 1.31 & 1.56 \\
\hline 2 & 0.5 & 0.88 & 1.88 \\
\hline 3 & 0.5 & 0.63 & 1.88 \\
\hline 4 & 0.63 & 1.31 & 0.5 \\
\hline 5 & 0.44 & 0.94 & 2.63 \\
\hline 6 & 0.63 & 1.44 & 2.81 \\
\hline 7 & 0.50 & 1.13 & 1.75 \\
\hline 8 & 0.44 & 1.38 & 1.56 \\
\hline Means (Std. Error) & $0.50(0.09)$ & $1.13(0.29)$ & $1.82(0.71)$ \\
\hline
\end{tabular}

Table III.2: Discrimination thresholds for each subject by TTC reference for the truck model (Experiment 1). These values were computed by subject as the average of the last four reversals. Also shown are the population mean and standard error of the mean.

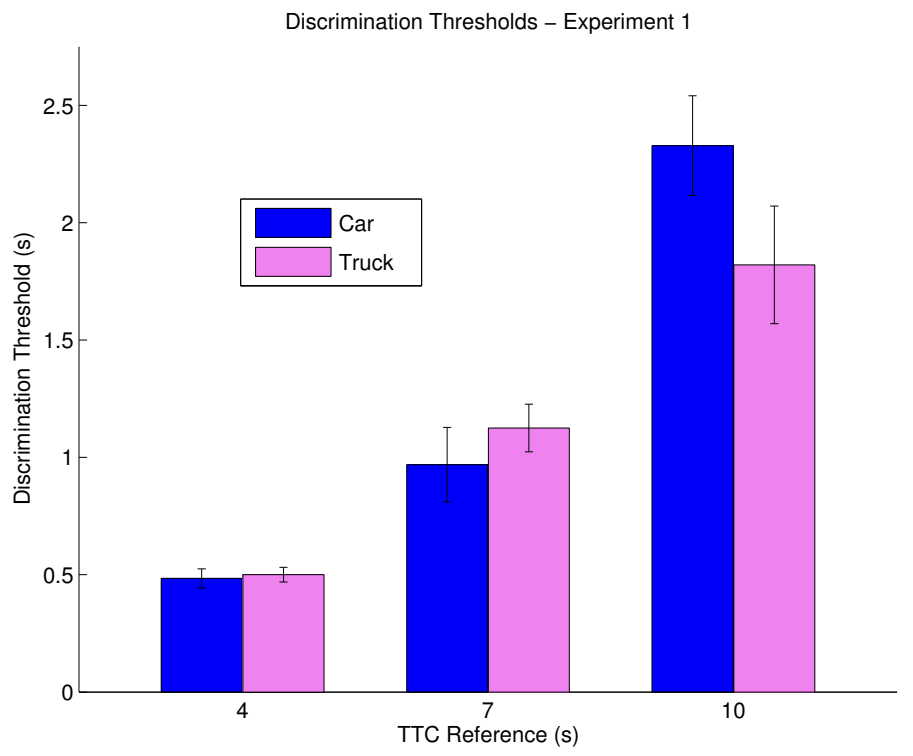

Figure III.5: The mean thresholds for the vehicle model discrimination task (Experiment 1). The means were determined by averaging the final four reversals for TTC reference times of $4 \mathrm{~s}, 7 \mathrm{~s}$, and $10 \mathrm{~s}$ for each vehicle model. Error bars show standard errors of the mean. 


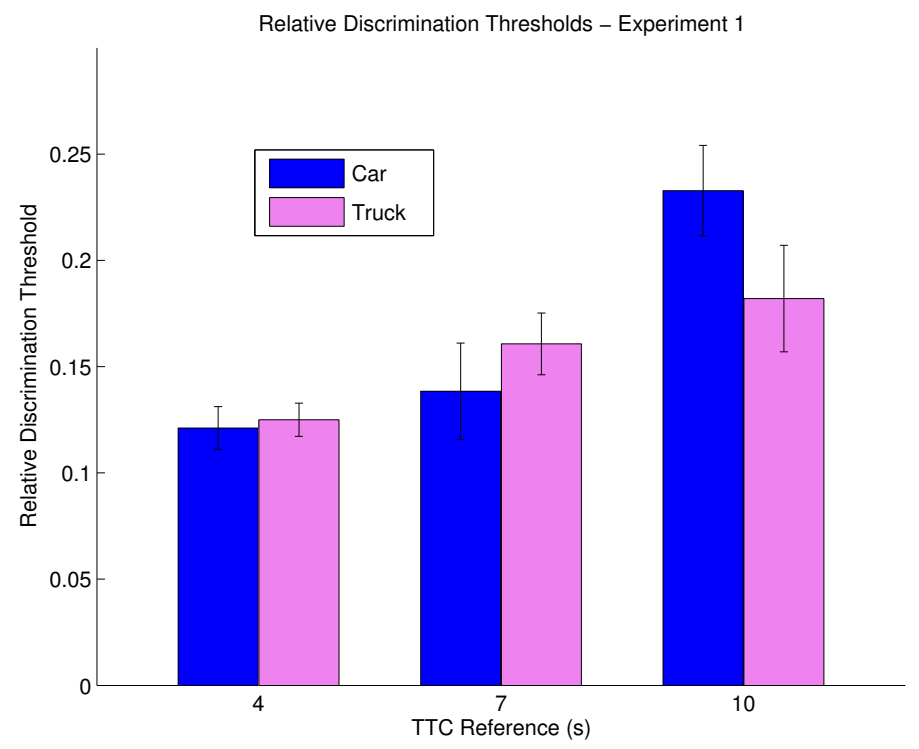

Figure III.6: The mean relative thresholds for the vehicle model discrimination task (Experiment 1) as functions of the TTC reference values. Error bars show standard errors of the mean. 


\section{CHAPTER IV}

\section{EXPERIMENT 2: EFFECT OF PEDESTRIAN VIEWPOINT}

This chapter presents an experiment to examine the influence of pedestrian viewpoint on TTC judgments. The task and its results are described.

\section{IV.1 Method}

In Experiment 1, we used graphical vehicles moving along a direct approach path toward the viewer to provide a basis for our time-to-contact pedestrian studies. However, pedestrians must make a decision whether to cross a street when they are observing a moving vehicle from the side of the road. For a typical two-lane road, the vehicle's trajectory of approach depends on which side of the road the pedestrian is standing. Therefore, we conducted an experiment to compare a head-on approach trajectory with the bypass trajectories that pedestrians observe from either side of the road.

\section{IV.1.1 Subjects}

Eight subjects, five males and three females, completed this experiment. Subjects' ages ranged from 17-30. All had normal or corrected-to-normal vision and were compensated for their participation. One subject also participated in Experiment 1.

\section{IV.1.2 Materials and Apparatus}

Three pedestrian viewpoints were compared: from the side of the road with the vehicle coming from the observer's left, from the side of the road with the vehicle coming from the observer's right, and a direct approach, i.e., the observer is in the middle of the vehicle lane. The viewpoints from the side of the road were generated by maximizing the view of the entire road. We used three vehicle models: a sedan, a semi-trailer truck, and an SUV. The SUV was added to give a vehicle whose size was midway between the sedan and the 


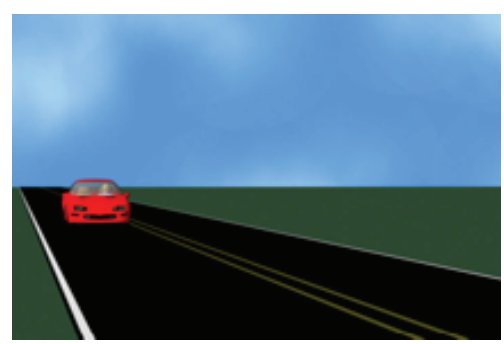

(a) Oncoming Left - Sedan

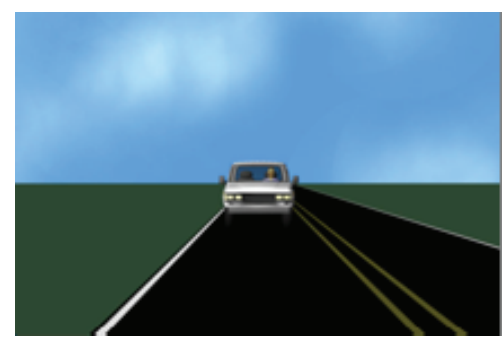

(b) Middle - SUV

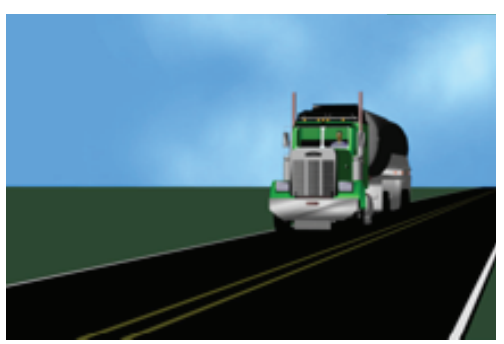

(a) Oncoming Right - Truck

Figure IV.1: Examples of the first image in a 2s video for the the sedan, SUV, and truck from various viewpoints with a TTC of $4 \mathrm{~s}$ and a velocity of $20 \mathrm{mph}$.

truck. Example images are shown in Figure IV.1.

For each combination of viewpoint and vehicle model, $2 \mathrm{~s}$ videos were generated for TTCs from $2 \mathrm{~s}$ to $14 \mathrm{~s}$ at $0.25 \mathrm{~s}$ intervals. A given TTC, viewpoint, and vehicle model were used in five separate videos, each with one of five vehicle velocities: 10, 15, 20, 25, and 30 mph. The same desktop setup as Experiment 1 was used in this task.

\section{IV.1.3 Procedure}

A discrimination task similar to Experiment 1 was conducted to compare the three viewpoints described above. Each trial compared two videos, both showing one of the three viewpoints. The order of viewpoints presented was randomly selected. Subjects were instructed to imagine they were standing at various positions along a crosswalk, depending upon the viewpoint shown. For a given trial, they were asked to determine which vehicle would reach the crosswalk first.

Each pair of videos had a video with a TTC reference value, and another video with a TTC that was greater or less by the staircase procedure described in Experiment 1. A velocity $(10,15,20,25$, or $30 \mathrm{mph})$ was randomly selected for each video. We did not control for effects of velocity. Since we found in Experiment 1 that vehicle model is not significantly influential on TTC judgments, we randomly selected a model for a given trial. We also randomized the model in order to replicate the effect in Experiment 1. Even though 


\begin{tabular}{|c|l|l|l|}
\hline & \multicolumn{3}{|c|}{ TTC Ref. $=6 \mathrm{~s}$} \\
\hline Subject & Oncoming Left & \multicolumn{1}{|c|}{ Middle } & Oncoming Right \\
\hline 1 & 1.38 & 1.75 & 0.88 \\
\hline 2 & 1.19 & 0.56 & 1.25 \\
\hline 3 & 1.00 & 0.81 & 1.00 \\
\hline 4 & 0.50 & 1.38 & 1.31 \\
\hline 5 & 1.56 & 2.38 & 3.25 \\
\hline 6 & 1.50 & 0.50 & 1.69 \\
\hline 7 & 1.50 & 0.63 & 0.69 \\
\hline 8 & 0.75 & 1.56 & 0.44 \\
\hline Means (Std. Error) & $1.17(0.14)$ & $1.20(0.24)$ & $1.31(0.31)$ \\
\hline
\end{tabular}

Table IV.1: Discrimination thresholds for each viewpoint for the 6s TTC reference (Experiment 2). These values were computed by subject as the average of the last four reversals. Also shown are the population mean and standard error of the mean.

we did not control the number of trials showing each vehicle model, the number of trials for each type would be approximately the same due to the random selection process used for each trial. We used two TTC references, 6s and 10s. The experiment was divided into two sections: one for the 6s TTC reference and the other for the 10s TTC reference. Only two reference values were used in order to keep the duration of the experiment within a reasonable limit while still allowing the observance of how judgment accuracy compares for short- and long-range TTCs. Separate threshold estimates were obtained for the reference values of $6 \mathrm{~s}$ and $10 \mathrm{~s}$ for each viewpoint. That is, there were six threshold procedures, one for each (Viewpoint, TTC Reference) pair. The experiment ended when the subjects reached 10 reversals or gave seven correct answers in a row for the lowest TTC difference, $0.25 \mathrm{~s}$, for the three viewpoints for each section. The number of trials was approximately the same across these conditions. The average number of trials per threshold procedure was 34. The order of the TTC reference sections was counterbalanced across subjects. Subjects were given a written set of instructions for the experiment and shown two practice trials. 


\section{IV.2 Results}

Tables IV.1 and IV.2 show the discrimination thresholds for each participant that were computed as the average of the TTC differences at the last four reversals for each viewpoint and TTC reference. These are also shown in Figure IV.2. A trend analysis of variance was performed with both TTC Reference $(6,10)$ and Viewpoint as repeated-measures factors. There was a significant effect of TTC Reference, $F(1,14)=13.789, p=.008$. Neither Viewpoint nor the interaction effect was significant.

Discrimination thresholds were also converted from seconds to a proportion of the reference TTC to assess whether discrimination ability is a constant proportion of the reference value of TTC. Mean relative thresholds for the vehicle coming from the observer's left were 0.20 and 0.18 of the 6 and 10 reference values, respectively. Mean relative thresholds for the direct approach were 0.20 and 0.21 of the 6 and 10 reference values, respectively. Mean relative thresholds for the vehicle coming from the observer's right were 0.22 and 0.18 of the 6 and 10 reference values, respectively. These relative thresholds were analyzed with the same design including TTC Reference and Viewpoint. No significant differences were found. The effect of vehicle model was also examined by looking at whether the vehicle model tended to result in more correct or more incorrect answers or an equal amount of both. We found that discrimination answers did not significantly depend on the vehicle model shown. These results are consistent with those found in Experiment 1. We did not examine effects of repeated subjects.

In conclusion, we found that the vehicle trajectory of approach in a pedestrian-crossing situation does not strongly influence TTC judgments. We also observed that discrimination accuracy decreased absolutely for longer TTCs across viewpoints. In addition, in agreement with Experiment 1, we did not find an effect of vehicle model. 


\begin{tabular}{|c|l|l|l|}
\hline & \multicolumn{3}{|c|}{ TTC Ref. $=10 \mathrm{~s}$} \\
\hline Subject & Oncoming Left & Middle & Oncoming Right \\
\hline 1 & 2.50 & 2.13 & 2.13 \\
\hline 2 & 1.38 & 3.00 & 1.38 \\
\hline 3 & 1.63 & 1.06 & 1.13 \\
\hline 4 & 2.06 & 1.19 & 1.31 \\
\hline 5 & 2.25 & 1.69 & 2.50 \\
\hline 6 & 0.88 & 2.13 & 2.13 \\
\hline 7 & 2.13 & 2.44 & 2.19 \\
\hline 8 & 1.56 & 3.06 & 1.44 \\
\hline Means (Std. Error) & $1.80(0.19)$ & $2.09(0.26)$ & $1.77(0.18)$ \\
\hline
\end{tabular}

Table IV.2: Discrimination thresholds for each viewpoint for the 10s TTC reference (Experiment 2). These values were computed by subject as the average of the last four reversals. Also shown are the population mean and standard error of the mean.

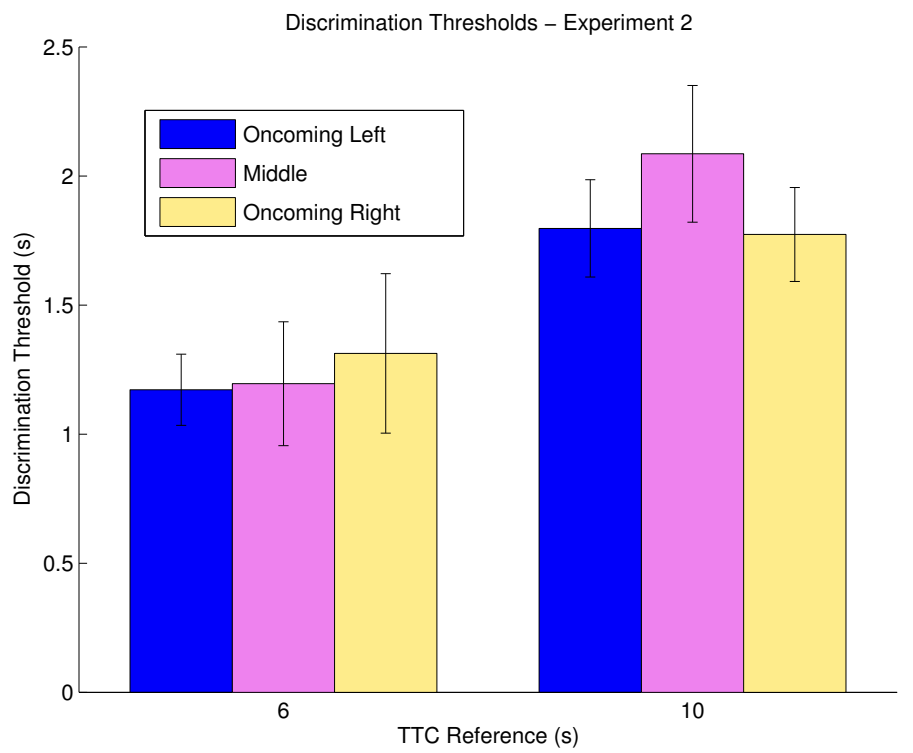

Figure IV.2: The mean thresholds for the viewpoint discrimination task (Experiment 2). The means were determined by averaging the final four reversals for TTC reference times of $6 \mathrm{~s}$ and $10 \mathrm{~s}$ for each viewpoint. Error bars show standard errors of the mean. 


\section{CHAPTER V}

\section{EXPERIMENT 3: SHORT- AND LONG-RANGE TTC JUDGMENTS}

This chapter presents an experiment to examine the influence of pedestrian viewpoint on TTC judgments. Two tasks and their results are described.

\section{V.1 Method}

Results from Experiment 1 and Experiment 2 indicate that neither vehicle model nor pedestrian viewpoint appear to affect TTC judgments. Next we sought to focus on the difference in TTC judgments for short- and long-range TTCs. Since bypass trajectories used in typical pedestrian-crossing scenarios are not significantly different than head-on trajectories, we decided to use the head-on trajectory employed by many TTC studies, e.g., [Schiff and Detwiler 1979]. We also wanted to compare the relationship between TTC discrimination ability using a relative judgment task and TTC estimation ability using a prediction-motion task. Therefore, we conducted a study with these two types of tasks. Both experiments were performed in a desktop environment and utilized the same setup as in Experiments 1 and 2. Subjects were given a set of written instructions for each experiment and shown a couple of practice trials for each task. The order of experiments was counterbalanced across subjects.

\section{V.1.1 Subjects}

Eight subjects, five males and three females, completed both tasks in this study. Subjects' ages ranged from 24-29. All had normal or corrected-to-normal vision and were compensated for their participation. One subject also participated in Experiment 2. 


\section{V.1.2 Procedures for Experiment 3a: Discrimination Task}

A discrimination task similar to Experiment 1 was conducted to compare short-range vs. long-range TTC judgments. Three reference TTC values were used: $4 \mathrm{~s}, 7 \mathrm{~s}$, and 10s. The velocities $(10,15,20,25$, and $30 \mathrm{mph})$ and TTC ranges (2s-14s) from Experiment 1 were used. Since results from Experiment 1 indicated that vehicle model is not significant, only the model of the sedan was used for this task. The adaptive staircase procedure from Experiment 1 was used. A constant velocity $(10,15,20,25$, or $30 \mathrm{mph})$ was randomly selected for each video. We did not control for effects of velocity. Separate threshold estimates were obtained for the reference values of $4 \mathrm{~s}, 7 \mathrm{~s}$, and 10s. The experiment ended when the subjects reached 10 reversals or gave seven correct answers in a row for the lowest TTC difference, $0.25 \mathrm{~s}$, for the three reference TTCs. The number of trials was approximately the same across these conditions. The average number of trials per threshold procedure was 34. Subjects were given a written set of instructions and shown two practice trials.

\section{V.1.3 Procedures for Experiment 3b: Estimation Task}

In Experiment 3b, the estimation task, participants were shown videos of the same car with TTC references of $4 \mathrm{~s}, 7 \mathrm{~s}$, or $10 \mathrm{~s}$, followed by a blank screen. For each $2 \mathrm{~s}$ video, subjects were asked to press a key at the moment they thought the car would reach them. 30 trials, 10 for each TTC reference, were presented to each subject in a random order. Each video was randomly assigned one of five vehicle velocities: $10,15,20,25$, or $30 \mathrm{mph}$. Subjects were given a written set of instructions and shown two practice trials.

\section{V.2 Results}

Table V.1 and Figure V.1 show the discrimination thresholds that were computed as the average of the TTC differences at the last four reversals. A trend analysis of variance was performed with TTC Reference $(4,7,10)$ as the repeated-measures factor and Task Order (Experiment 1 first and then Experiment 2 or vice versa) as the between-groups 


\begin{tabular}{|c|l|l|l|}
\hline Subject & TTC Ref. $=4 \mathrm{~s}$ & TTC Ref. $=$ 7s & TTC Ref. = 10s \\
\hline 1 & 0.38 & 0.38 & 2.25 \\
\hline 2 & 0.44 & 1.63 & 2.63 \\
\hline 3 & 0.50 & 0.81 & 1.44 \\
\hline 4 & 0.38 & 2.00 & 1.56 \\
\hline 5 & 0.50 & 2.00 & 2.13 \\
\hline 6 & 0.63 & 0.75 & 1.63 \\
\hline 7 & 0.69 & 0.44 & 1.69 \\
\hline 8 & 0.69 & 1.38 & 1.25 \\
\hline Means (Std. Error) & $0.52(0.05)$ & $1.17(0.24)$ & $1.82(0.16)$ \\
\hline
\end{tabular}

Table V.1: Discrimination thresholds for each subject by TTC reference for the discrimination task (Experiment 3a). These values were computed by subject as the average of the last four reversals. Also shown are the population mean and standard error of the mean.

factor. There was a significant linear trend for TTC References, $F(1,6)=50.717, p<.001$. Neither Task Order nor the interaction effect was significant.

Discrimination thresholds were also converted from seconds to a proportion of the reference TTC to assess whether discrimination ability is a constant proportion of the reference value of TTC. Mean relative thresholds were $0.13,0.17$, and 0.18 of the 4,7 , and 10 reference values, respectively, and are shown in Figure V.2. These relative thresholds were analyzed with the same design including TTC Reference and Task Order. Again, only the linear trend for TTC Reference was significant, $F(1,6)=6.005, p<.05$. We did not look at effects of repeated subjects.

For the estimation task, the means for each subject for each TTC reference value, along with the standard errors and the slope of the regression line fitted through their means are shown in Table V.2. Their means and standard errors are also shown in Figure V.3. On average, there was a consistent bias towards overestimating the TTC. This result is somewhat surprising, since one might guess that subjects would be biased to not waiting very long and underestimating the TTC, which has been found in [Schiff and Detwiler 1979; Cavallo and Laurent 1988]. In terms of constant and variable errors, the variable 


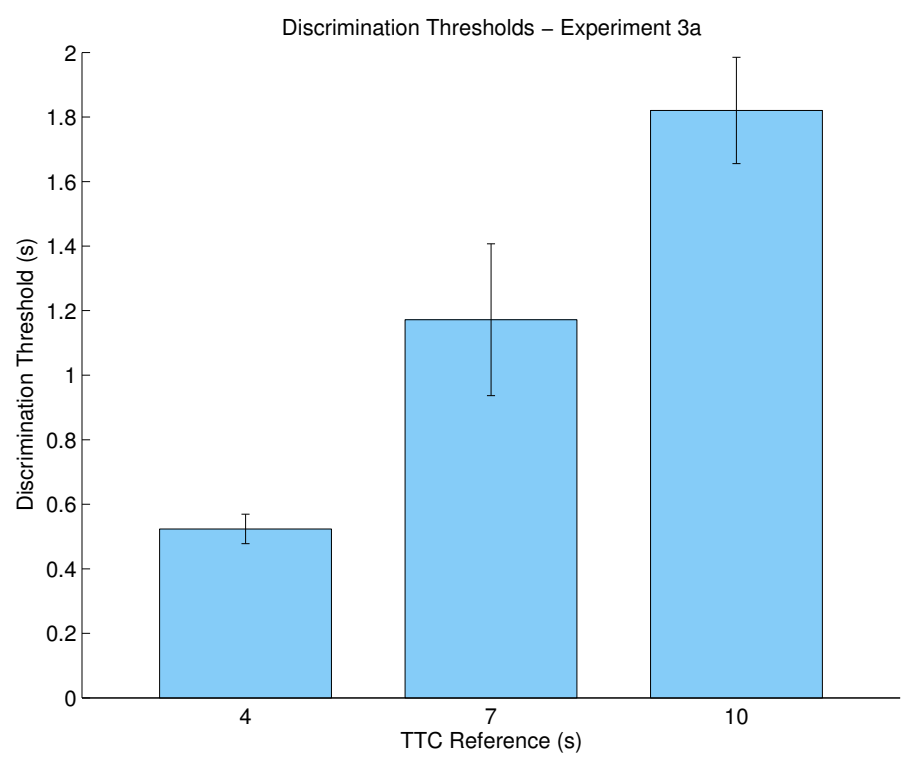

Figure V.1: The mean thresholds for the discrimination task (Experiment 3a). The means were determined by averaging the final four reversals for TTC reference times of $4 \mathrm{~s}, 7 \mathrm{~s}$, and 10s. Error bars show standard errors of the mean.

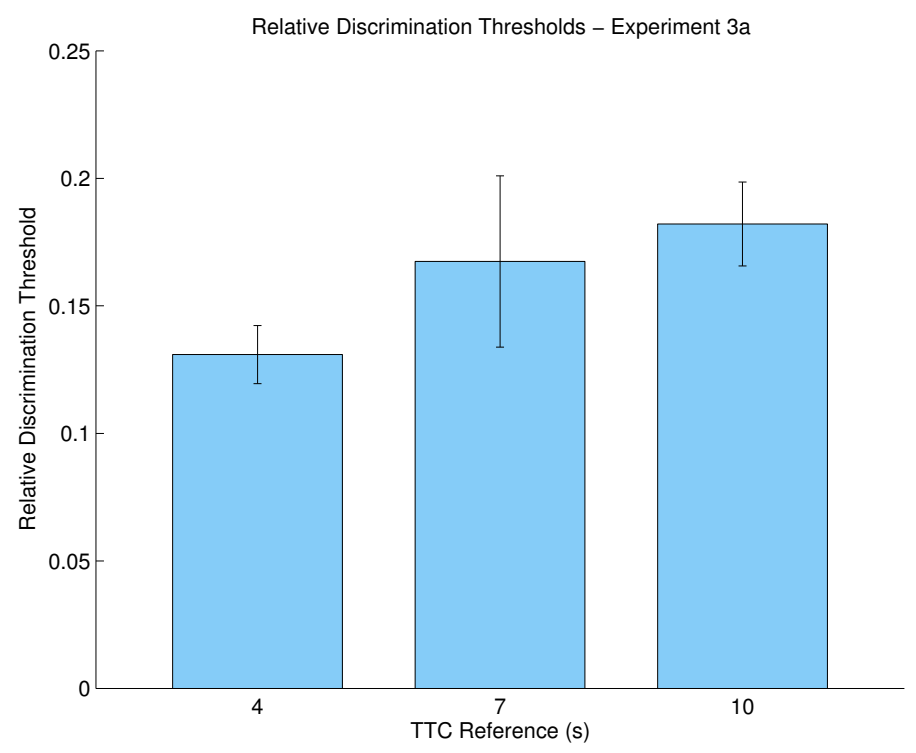

Figure V.2: The mean relative thresholds for the discrimination task (Experiment 3a) as functions of the TTC reference values. Error bars show standard errors of the mean. 
error was the largest component of error for seven subjects. We analyzed variable errors for systematic differences between errors across TTC reference values but found that it was not a significant component of the variable error. Thus, subjects were able to systematically scale their responses according to the reference values, as is evident by the slopes of the regression lines, shown in Table V.2.

Overall, subjects were accurate at estimating TTC. Absolute error was greater for larger reference values, which indicates that subjects were less accurate at larger ranges of TTCs. To examine accuracy relative to the TTC reference value, the mean errors were converted to a proportion of the reference values, similar to the method used for the discrimination thresholds. These errors were $6.7 \%, 7.6 \%, 15 \%$ for $4 \mathrm{~s}, 7 \mathrm{~s}$, and $10 \mathrm{~s}$ respectively, and the overall differences between them were not statistically significant. No direct correlation was found between participants' accuracy for the discrimination task with that for the estimation task.

In conclusion, we found that in discrimination and estimation accuracy declined for longer TTCs, and we did not observe any correlation between performance for the two tasks. 


\begin{tabular}{|l|l|l|r|r|}
\hline Subject & \multicolumn{3}{|c|}{ Mean (Std. Error) } & Slope \\
\hline & TTC Ref. $=4 \mathrm{~s}$ & TTC Ref. $=7 \mathrm{~s}$ & \multicolumn{1}{|c|}{ TTC Ref. $=10 \mathrm{~s}$} & \\
\hline 1 & $4.70(0.32)$ & $9.69(0.62)$ & $17.79(1.38)$ & 2.18 \\
\hline 2 & $4.04(0.16)$ & $7.90(0.32)$ & $11.45(0.85)$ & 1.23 \\
\hline 3 & $3.41(0.14)$ & $4.98(0.21)$ & $6.77(0.29)$ & 0.56 \\
\hline 4 & $3.56(0.34)$ & $5.98(0.35)$ & $8.15(0.32)$ & 0.76 \\
\hline 5 & $5.75(0.43)$ & $9.41(0.61)$ & $16.55(1.62)$ & 1.80 \\
\hline 6 & $3.86(0.24)$ & $6.58(0.27)$ & $8.40(0.31)$ & 0.76 \\
\hline 7 & $4.46(0.48)$ & $8.44(0.77)$ & $12.12(0.84)$ & 1.28 \\
\hline 8 & $4.36(0.48)$ & $7.29(0.68)$ & $11.02(1.28)$ & 1.11 \\
\hline Means & 4.26 & 7.53 & 11.53 & 1.21 \\
\hline
\end{tabular}

Table V.2: Results from Experiment 3b. For each subject their mean estimated TTC for each reference value with their standard error over 10 trials shown in parentheses. Additionally, for each subject the slope of a linear regression of their data is shown in the final column. The total mean estimated value and average slope are shown in the bottom row.

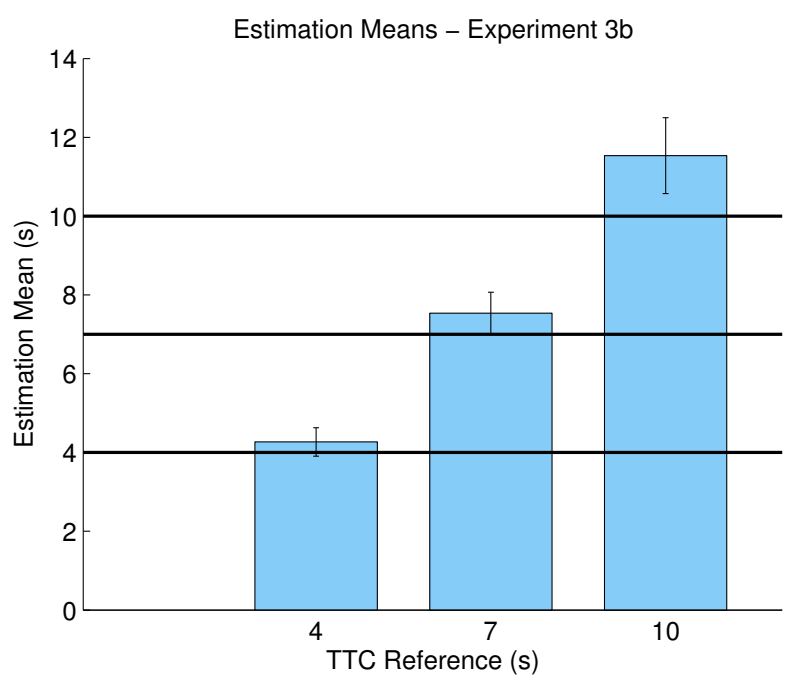

Figure V.3: The mean values for the estimation task (Experiment 3b). The means were determined by averaging each subject's mean estimated TTC for each reference value. Error bars show standard errors of the mean. The black solid lines show the correct estimations. 


\section{CHAPTER VI}

\section{EXPERIMENT 4: EFFECT OF IMMERSIVE VIEWING}

This chapter presents an experiment to examine the influence of immersive viewing on TTC judgments. The task and its results are described.

\section{VI.1 Method}

In Experiments 1-3, a desktop environment was used to learn about people's accuracy at judging time-to-contact. Although a desktop setup has been used in many TTC studies, it is limited in its ability to give subjects a sense of immersion. Also, subjects are not able to track the moving vehicle with their heads when viewing animations on a monitor. In the real world, people probably adapt their viewpoint according to the position of the approaching vehicle when observing its path from the side of the road. An immersive virtual environment allows for this self-motion and may feel more similar to a real-world environment. Experiment 4 examines these influences on TTC judgments.

\section{VI.1.1 Subjects}

Eight subjects, five females and three males, completed this experiment. Subjects' ages ranged from 20-30. All had normal or corrected-to-normal vision and were compensated for their participation. Two subjects also participated in Experiment 1 and one subject in Experiment 3.

\section{VI.1.2 Materials and Apparatus}

The virtual environment consisted of an infinite two-lane road with a crosswalk on top of an infinite green ground plane with a sky blue background. The environment looked the same as the first image shown in Figure IV.1. All objects in the scene had realistic dimensions. The virtual world was viewed through a full color stereo eMagin Z800 3DVisor with a full 
resolution of $800 \times 480$, a field of view of 40 degrees diagonally, and a frame rate of 60 Hz. A subject's rotational movements were updated using an InterSense IS-900 tracker. A subject's translational movements were updated using two optical tracking cameras with an accuracy of $<0.5 \mathrm{~cm}$ over $3 \times 3 \times 3 \mathrm{~m}$ volume and an update rate of $60 \mathrm{~Hz}$. A joystick was used for subject input because it allowed subjects to easily enter data while wearing the head-mounted display. The joystick used in this experiment was the Logitech Attack 3.

\section{VI.1.3 Procedure}

A discrimination task similar to Experiment 3 was conducted to test the effect of selfmotion on TTC judgments in an immersive virtual environment. The discrimination task employed the same adaptive methodology as the task described in Experiment 2. The two TTC references used were $4 \mathrm{~s}$ and $7 \mathrm{~s}$ as opposed to the $6 \mathrm{~s}$ and $10 \mathrm{~s}$ references in Experiment 2. These shorter references were adopted due to the limited graphical capabilities of our head-mounted display system. At very long TTCs, the image was not clear. Only the sedan model was shown in this experiment since Experiments 1 and 2 showed that the model effect was negligible on TTC judgments. For each trial, subjects were shown a pair of $2 \mathrm{~s}$ animations, where the car appeared at a given starting distance on the road, moved forward at a randomly selected velocity $(10,15,20,25$, or $30 \mathrm{mph})$, and disappeared from view after $2 \mathrm{~s}$. Buttons on a joystick were used by the subject to select which animated car would reach the crosswalk first. We did not control for effects of velocity.

The experiment was composed of two sections: one showing videos of short-range TTCs around $4 \mathrm{~s}$ and the other showing videos of longer-range TTCs around 7s. Separate threshold estimates were obtained for the reference values of $4 \mathrm{~s}$ and $7 \mathrm{~s}$ for each viewpoint. That is, there were six threshold procedures, one for each (Viewpoint, TTC Reference) pair. The number of trials was approximately the same across these conditions. The aver-

age number of trials per threshold procedure was 34. Subjects were given a written set of instructions for the experiment and shown two practice trials. Subjects were also given a 
15-minute break between sections of the experiment. The order of sections was counterbalanced across subjects.

\section{VI.2 Results}

Tables VI.1 and VI.2 show the discrimination thresholds for each participant that were computed as the average of the TTC differences at the last four reversals for each viewpoint and TTC reference. These are also shown in Figure VI.1. A trend analysis of variance was performed with both TTC Reference $(4,7)$ and Viewpoint as repeated-measures factors. There was a significant effect of TTC Reference, $F(1,14)=116.354, p<.001$. Neither Viewpoint nor the interaction effect was significant. Therefore, as in Experiment 2, we conclude that the vehicle trajectory of approach in a pedestrian-crossing situation does not strongly influence TTC judgments.

Discrimination thresholds were also converted from seconds to a proportion of the reference TTC to assess whether discrimination ability is a constant proportion of the reference value of TTC. Mean relative thresholds for the vehicle coming from the observer's left were 0.20 and 0.22 of the 4 and 7 reference values, respectively. Mean relative thresholds for the direct approach were 0.15 and 0.24 of the 4 and 7 reference values, respectively. Mean relative thresholds for the vehicle coming from the observer's right were 0.14 and 0.23 of the 4 and 7 reference values, respectively. These relative thresholds are shown in Figure VI.2. They were analyzed with the same design including TTC Reference and Viewpoint. Again, only the linear trend for TTC Reference was significant, $F(1,14)=9.498, p=.018$. Neither Viewpoint nor the interaction effect were significant. We did not look at effects of repeated subjects.

In conclusion, we did not find any effect of viewpoint, which indicates that self-motion does not strongly affect TTC judgments. The lack of viewpoint effect is in agreement with results found in Experiment 2 for a desktop environment. Also, we found that discrimination accuracy decreased absolutely and relatively for longer TTCs across viewpoints. 


\begin{tabular}{|c|l|l|l|}
\hline & \multicolumn{3}{|c|}{ TTC Ref. $=4 \mathrm{~s}$} \\
\hline Subject & Oncoming Left & \multicolumn{1}{|c|}{ Middle } & Oncoming Right \\
\hline 1 & 0.50 & 0.25 & 0.56 \\
\hline 2 & 0.69 & 0.56 & 0.5 \\
\hline 3 & 0.75 & 0.44 & 0.56 \\
\hline 4 & 0.75 & 0.94 & 0.25 \\
\hline 5 & 0.88 & 0.44 & 0.56 \\
\hline 6 & 0.69 & 0.5 & 0.38 \\
\hline 7 & 0.88 & 0.25 & 0.25 \\
\hline 8 & 1.31 & 1.38 & 1.38 \\
\hline Means (Std. Error) & $0.80(0.08)$ & $0.59(0.14)$ & $0.55(0.13)$ \\
\hline
\end{tabular}

Table VI.1: Discrimination thresholds for each viewpoint for the 4s TTC reference (Experiment 4). These values were computed by subject as the average of the last four reversals. Also shown are the population mean and standard error of the mean.

\begin{tabular}{|c|l|l|l|}
\hline & \multicolumn{3}{|c|}{ TTC Ref. $=7 \mathrm{~s}$} \\
\hline Subject & Oncoming Left & Middle & Oncoming Right \\
\hline 1 & 2.25 & 1.44 & 1.88 \\
\hline 2 & 1.00 & 2.13 & 1.63 \\
\hline 3 & 1.63 & 2.31 & 0.88 \\
\hline 4 & 1.44 & 1.38 & 1.13 \\
\hline 5 & 1.88 & 1.00 & 2.06 \\
\hline 6 & 1.75 & 0.81 & 2.06 \\
\hline 7 & 1.63 & 1.06 & 1.19 \\
\hline 8 & 0.81 & 3.06 & 2.06 \\
\hline Means (Std. Error) & $1.54(0.16)$ & $1.65(0.28)$ & $1.61(0.17)$ \\
\hline
\end{tabular}

Table VI.2: Discrimination thresholds for each viewpoint for the 7s TTC reference (Experiment 4). These values were computed by subject as the average of the last four reversals. Also shown are the population mean and standard error of the mean. 


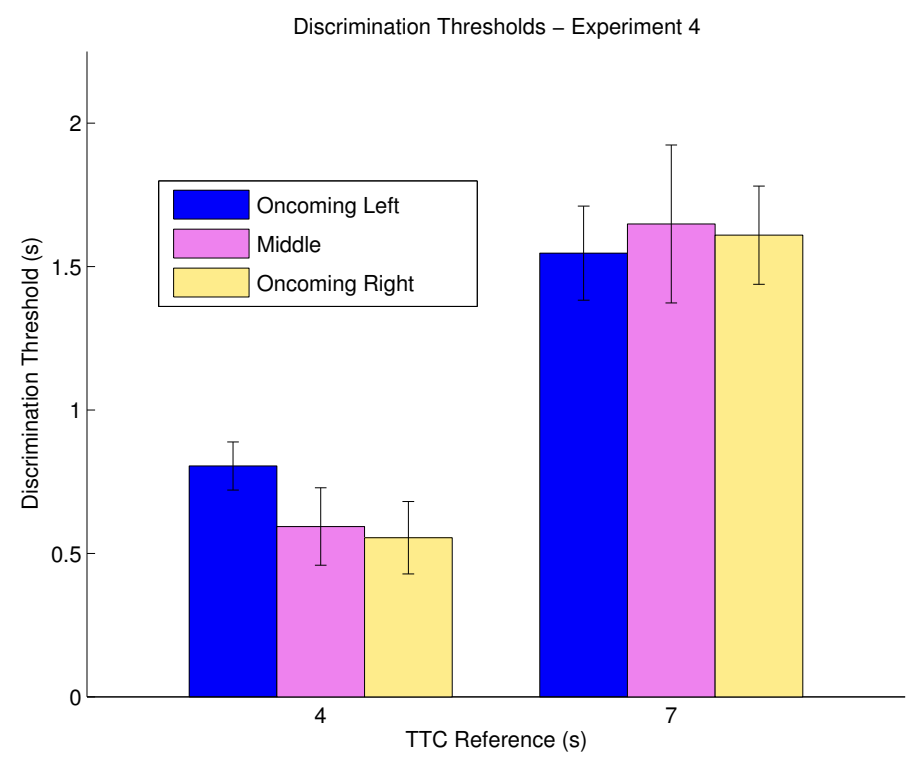

Figure VI.1: The mean thresholds for the viewpoint discrimination task in the immersive virtual environment (Experiment 4). The means were determined by averaging the final four reversals for TTC reference times of $4 \mathrm{~s}$ and $7 \mathrm{~s}$ for each viewpoint. Error bars show standard errors of the mean.

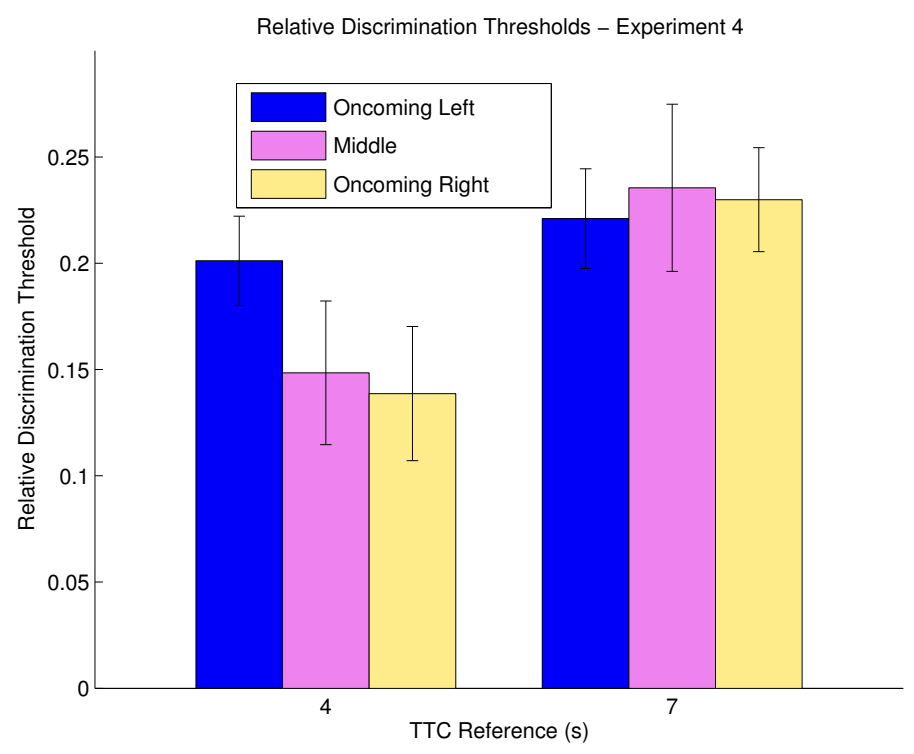

Figure VI.2: The mean relative thresholds for the vehicle model discrimination task in the immersive virtual environment (Experiment 4) as functions of the TTC reference values. Error bars show standard errors of the mean. 


\section{CHAPTER VII}

\section{DISCUSSION}

This chapter discusses the results of the four experiments presented in this thesis. Future directions for this research are also described.

This thesis presents four experiments that were conducted to assess time-to-contact (TTC) in a vehicular setting and in both desktop and immersive virtual environments. One goal was to assess how perceptions of oncoming traffic in virtual environments correlated with prior work on TTC, which typically assesses TTC using simpler stimuli. Our work extended the prior work into novel situations that pedestrians typically encounter in crossing streets. In street crossings not mediated by a crossing signal, people generally estimate arrival times of vehicles, and then choose whether to cross the street based on those observations.

Our experiments examined the effect of vehicle model, pedestrian viewpoint, short- and long-range TTCs, and presentation mode on TTC judgments. In our first experiment, we found that the type of vehicle model was not significant in subjects' ability to discriminate TTC. This finding is in agreement with some prior work [Schiff and Detwiler 1979; Kim and Grocki 2006], although Caird and Hancock [1994] observed an effect of vehicle model in an estimation task using a driving simulator. This difference may mean that discrimination ability is cognitively separate from estimation ability.

Our second experiment showed that bypass views of vehicles, views generally observed by pedestrians when watching an approaching vehicle from the side of a road, did not result in significantly different discrimination than head-on approaches. When the bypass view occurs on the near (left) side of the road, our results are consistent with those found in [Schiff and Oldak 1990], who concluded that bypass trajectories with angles less than $10^{\circ}$ at the vehicle vanishing distance are not judged differently than head-on trajectories. 
Our results extend that angle for situations occurring on the far (right) side of the road, where the bypass angle is a maximum of $29.4^{\circ}$ for vehicles at a TTC of 4 s and a velocity of $10 \mathrm{mph}$.

Our third experiment shows that subjects can discriminate TTCs on the order of $15 \%$ of the reference TTC for approaching vehicles in the speed and distance range that pedestrians typically encounter. We found that the level of performance, however, declines significantly with longer TTCs. These longer TTCs are those in which pedestrians are typically making crossing decisions [Guth et al. 2005]. Our thresholds for short TTCs were consistent with thresholds determined by other research studies. Additionally, people's level of performance declines absolutely and relatively with longer TTCs.

This experiment also contained an estimation task. People were within 10\% (overall) in their absolute judgment of TTC. Surprisingly, people did not consistently underestimate TTC in our results, a finding contrary to the trend reported in prior work [Schiff and Detwiler 1979; Cavallo and Laurent 1988; Gray and Regan 1999]. This result is surprising since one might guess that the experiment method would bias subjects to underestimate TTC. However, our result is consistent with studies examining real-world pedestrian situations at traffic roundabouts, which have found that pedestrians with normal vision leave small margins of safety of only a second or two when crossing the street [Guth et al. 2005]. These small gap affordances may reflect a trend to overestimate TTC.

Finally, immersive viewing in an HMD does not give significant differences in TTC judgments compared to those made in a desktop environment. Consistent with our second experiment, we found that pedestrian viewpoint does not make a difference in TTC discrimination ability. For viewpoints from the side of the road, immersive viewing allowed subjects to track the oncoming vehicle with head movement. Because TTC judgments made at the side of the road were not significantly different from judgments made in the middle of the road, we conclude that self-motion does not improve judgment accuracy.

In general, people seem to be using strategies similar to those that pedestrians might 
use to make street-crossing decisions. In some post-experiment interviews, subjects reported being aware that both the speed and the apparent size of the car varied, and that they could not rely on only one cue to make their choice. Our results indicate that similar decision-making processes reported in the literature [Ashmead et al. 2005] for pedestrian crossing are occurring in our laboratory settings using both desktop and immersive virtual environments. Experiments 1-4 all found a significant effect of reference time, meaning that judgment accuracy degrades for longer TTCs.

In particular, from our results, the side of the road that a pedestrian is on does not influence crossing decisions where the view of the oncoming vehicle(s) is unobstructed. Also, pedestrians who have difficulty crossing intersections may need to be more conservative in their judgments. Plumert et al. [2004] find this true especially for children on bicycles, who tend to experience greater difficulty in coordinating their actions with cars and therefore, have less time to spare between themselves and the approaching vehicles.

In the future, we would like to examine the effect of a detailed graphical environment that more closely resembles a real traffic crossing scene. Some subjects in the immersive viewing experiment commented that this virtual world was more compelling than the desktop environment. Perhaps adding buildings and other features would enhance the sense of immersion and yield TTC judgments that reflect the complexity of these decisions in the real world. A similar avenue of exploration involves designing an experimental task which demands that subjects make simulated crossing decisions. This research area would include adapting Plumert et al.'s [2004] bicycling task for applications of pedestrian crossings. 


\section{REFERENCES}

Ashmead, D. H., Guth, D., Wall, R. S., Long, R. G., And Ponchillia, P. E. 2005. Street crossing by sighted and blind pedestrians at a modern roundabout. J Trans Eng 131, 812-821.

Benguigui, N., Ripoll, H., AND BROderick, M. P. 2003. Time-to-contact estimation of accelerated stimuli is based on first-order information. J Exp Psychol Hum Percept Perform 29, 1083-1101.

Bootsma, R. J., AND CRAig, C. M. 2002. Global and local contributions to the optical specification of time to contact: observer sensitivity to composite tau. Perception 31, 901-924.

Bootsma, R. J., And Oudejans, R. R. D. 1993. Visual information about time-tocollision between two objects. J Exp Psychol Hum Percept Perform 19, 1041-1052.

CAIRD, J. K., AND HANCOCK, P. A. 1994. The perception of arrival time for different oncoming vehicles at an intersection. Ecological Psychology 6, 83-109.

Caljouw, S. R., van der Kamp, J., and Savelsbergh, G. J. P. 2004. Catching optical information for the regulation of timing. Exp Brain Res 155, 427-438.

Cavallo, V., And Laurent, M. 1988. Visual information and skill level in time-tocollision estimation. Perception 17, 623-632.

DeLucia, P. R., AND NovaK, J. B. 1997. Judgments of relative time-to-contact of more than two approaching objects: toward a method. Percept Psychophys 59, 913-928.

DeLuCiA, P. R. 2004. Time-to-contact judgments of an approaching object that is partially concealed by an occluder. J Exp Psychol Hum Percept Perform 30, 287-304.

GRAY, R., AND REGAN, D. 1999. Do monocular time-to-collision estimates necessarily involve perceived distance? Perception 28, 1257-1264.

Guth, D., Ashmead, D., Long, R., Wall, R., And Ponchillia, P. 2005. Blind and sighted pedestrians' judgments of gaps in traffic at roundabouts. Human Factors 47, 314-331.

Hecht, H., Kaiser, M. K., Savelsbergh, G. J., And VAn der Kamp, J. 2002. The impact of spatiotemporal sampling on time-to-contact judgments. Percept Psychophys $64,650-666$.

HeUER, H. 1993. Estimates of time to contact based on changing size and changing target vergence. Perception 22, 549-563.

IOWA Department of Transportation, 2005. Standard specifications with gs-01008 revisions, April. 
Kiefer, R., Flannagan, C., And Jerome, C. 2006. Time-to-collision judgments under realistic driving conditions. Human Factors 48, 334-345.

Kim, N., AND GROCKI, J. 2006. Multiple sources of information and time-to-contact judgments. Vis Res 46, 1946-1958.

LEE, D. N. 1976. A theory of visual control of braking based on information about time-to-collision. Perception 5, 437-459.

MAnser, M. P., AND HANCOCK, P. A. 1996. Influence of approach angle on estimates of time-to-contact. Ecological Psychology 8, 71-99.

Maxwell, S. E., And Delaney, H. D. 2004. Designing experiments and analyzing data. A model comparison perspective, second ed. Lawrence Erlbaum Associates, Mahwah, NJ, ch. 6, 243-274.

McLeOd, R. W., AND Ross, H. E. 1983. Optic-flow and cognitive factors in time-tocollision estimates. Perception 12, 417-423.

National Center for Statistics And AnAlysis, 2005. Traffic safety facts: 2005 data.

Pitt, R., Guyer, B., Hsieh, C. C., And MaleK, M. 1990. The severity of pedestrian injuries in children: an analysis of the pedestrian injury causation study. Accid Anal Prev $22,549-559$.

Plumert, J. M., Kearney, J. K., And Cremer, J. F. 2004. Children's perception of gap affordances: Bicylcing across traffic-filled intersections in an immersive virtual environment. Child Develop 75, 1243-1253.

Regan, D., And Hamstra, S. J. 1993. Dissociation of discrimination thresholds for time to contact and for rate of angular expansion. Vision Res 33, 447-462.

Schiff, W., AND Detwiler, M. L. 1979. Information used in judging impending collision. Perception 8, 647-658.

SCHIFF, W., AND OldAK, R. 1990. Accuracy of judging time to arrival: effects of modality, trajectory, and gender. J Exp Psychol Hum Percept Perform 16, 303-316.

Senot, P., Prevost, P., And McIntyre, J. 2003. Estimating time to contact and impact velocity when catching an accelerating object with the hand. J Exp Psychol Hum Percept Perform 29, 219-237.

Servos, P., And Goodale, M. A. 1998. Monocular and binocular control of human interceptive movements. Exp Brain Res 119, 92-102.

Sidaway, B., FAirweather, M., Sekiya, H., And McNitT-Gray, J. 1996. Time-tocollision estimation in a simulated driving task. Human Factors 38, 101-113. 
Smeets, J. B. J., Brenner, E., Trebuchet, S., And Mestre, D. R. 1996. Is judging time-to-contact based on 'tau'? Perception 25, 583-590.

TodD, J. T. 1981. Visual information about moving objects. J Exp Psychol Hum Percept Perform 7, 795-810.

Tresilian, J. R. 1993. Four questions of time to contact: a critical examination of research on interceptive timing. Perception 22, 653-680.

Tresilian, J. R. 1995. Perceptual and cognitive processes in time-to-contact estimation: analysis of prediction-motion and relative judgment tasks. Percept Psychophys 57, 231245.

von Hofsten, C., Rosengren, K., Pick, H. L., And Neely, G. 1992. The role of binocular information in ball catching. J Mot Behav 24, 328-338.

Wetherill, C. B., And LevitT, H. 1965. Sequential estimation of points on a psychometric function. British J Math Stat Psychol 18, 1-10. 